\title{
Development of a handheld diffuse optical breast cancer assessment probe
}

\author{
Majid Shokoufi and Farid Golnaraghi* \\ School of Mechatronic Systems Engineering \\ Simon Fraser University, 250-13450 102 Avenue \\ Surrey, Canada, BC V3T $0 A 3$ \\ *mfgolnar@sfu.ca
}

Received 10 June 2015

Accepted 15 September 2015

Published 30 October 2015

\begin{abstract}
Diffuse Optical Spectroscopy (DOS) is a promising non-invasive and non-ionizing technique for breast anomaly detection. In this study, we have developed a new handheld DOS probe to measure optical properties of breast tissue. In the proposed probe, the breast tissue is illuminated with four near infrared (NIR) wavelengths light emitting diodes (LED), which are encapsulated in a package (eLEDs), and two PIN photodiodes measure the intensity of the scattered photons at two different locations. The proposed technique of using eLEDs is introduced, in order to have a multi-wavelength pointed-beam illumination source instead of using the laser-coupled fiberoptic technique, which increases the complexity, size, and cost of the probe. Despite the fact that the proposed technique miniaturizes the probe and reduces the complexity of the DOS, the study proves that it is accurate and reliable in measuring optical properties of the tissue. The measurements are performed at the rate of $10 \mathrm{~Hz}$ which is suitable for dynamic measurement of biological activity, in-vivo. The multi-spectral evaluation algorithm is used to reconstruct four main absorber concentrations in the breast including oxy-hemoglobin $(\mathrm{cHb})$, deoxy-hemoglobin $(\mathrm{cHbO} 2)$, water $(\mathrm{cH} 2 \mathrm{O})$, fat (cFat), and average scattering coefficient of the medium, as well as concentration changes in $\mathrm{Hb}(\Delta \mathrm{cHb})$ and $\mathrm{HbO} 2(\Delta \mathrm{cHbO} 2)$. Although the probe is designed for breast cancer diagnosis, it can be used in a wide range of applications for both static and dynamic measurements such as functional brain imaging. A series of phantoms, comprised of Delrin ${ }^{\circledR}$, Intralipid ${ }^{\circledR}$, Pierce ${ }^{\mathrm{TM}}$ and Black ink, are used to verify performance of the device. The probe will be tested on human subjects, in-vivo, in the next phase.
\end{abstract}

Keywords: Breast cancer; diffuse optical spectroscopy; intrinsic optical contrast; hemoglobin concentration; optical breast phantom.

${ }^{*}$ Corresponding author.

This is an Open Access article published by World Scientific Publishing Company. It is distributed under the terms of the Creative Commons Attribution 3.0 (CC-BY) License. Further distribution of this work is permitted, provided the original work is properly cited. 


\section{Introduction}

Breast cancer is the uncontrolled growth of breast cells and is the second leading cause of cancer related death in women. In 2015, it was expected that about 25,168 and 231,840 women and men would be diagnozed with breast cancer in Canada and the USA, respectively. It was also anticipated that 5,000 women and 60 men in Canada, and 40,290 women and 430 men in the USA would die from breast cancer in 2015. ${ }^{1,2}$ Popular methods in breast cancer imaging are X-ray mammography, ultrasound, and magnetic resonance imaging (MRI), which X-ray mammography is the primary and golden standard for breast cancer screening. ${ }^{3}$ While mammography is the golden standard for breast cancer screening, it is only advised for women between the ages of 50 and $74^{4}$ due to its high false negative detection ratio for younger women ${ }^{3}$ and because of its potential health risks as a result of ionizing radiations (X-ray). ${ }^{5,6}$ The limitations of mammography have motivated researchers to develop alternative breast cancer detection systems such as diffuse optical spectroscopy (DOS), electrical impedance tomography (EIT), elastography. ${ }^{7}$ Over the past two decades near infraRed (NIR) DOS has been developed for biomedical applications and emerged as a promising technique for noninvasive cross-sectional imaging of highly scattering media, such as functional brain mapping and breast cancer diagnosis. ${ }^{7}$ Breast DOS measures optical properties of the biological tissues, primarily the absorption and the scattering coefficient in the NIR range at different locations of the breast. Three different technologies have been employed in DOT for the detection of breast cancer: (1) continuous wave $(\mathrm{CW})$, where the amplitude of the signal is measured, ${ }^{8}(2)$ frequency domain, in which the light sources are modulated, and the phase and amplitude of the scattered signal are measured, ${ }^{9}$ and (3) time domain (TD), in which ultra-short light pulses are used as the light source. ${ }^{8-10}$ There are a number of handheld DOS probes that have been developed for breast cancer diagnosis and monitoring. ${ }^{11-17}$ Source-detector separations on the probes are in the range of $0.5 \mathrm{~cm}$ to $3.5 \mathrm{~cm}$ and are positioned in reflectance geometry to measure the optical properties of the underneath tissue. ${ }^{11}$ They use a number of laser light sources or fiber coupled laser light sources in NIR range to determine the concentration of main absorbers in the breast tissue related to sensing anomalies including oxy-hemoglobin, deoxy-hemoglobin, water, and fat. In these probes, laser light sources are located $5 \mathrm{~mm}$ to $10 \mathrm{~mm}$ away from each other which reduces performance of the device to detect an anomaly tissue.

In this study, a handheld DOS probe is designed and implemented to measure optical properties of breast tissue. Here, we present the first prototype of the probe using multi-wavelengths encapsulated light emitting diodes (eLED) as a light source. The probe uses an LED package containing four NIR wavelengths, 690,750, 800 and $850 \mathrm{~nm}$, which are located $0.5 \mathrm{~mm}$ away from each other and act as a multi-wavelength point source. Using encapsulated multi-wavelengths LEDs miniaturizes the probe $(1.5 \mathrm{~cm} \times 4 \mathrm{~cm})$. The intensity of each LED is modulated with different radio frequencies, which allows the device to illuminate breast tissue with all four wavelengths, simultaneously. This technique improves the rejection of ambient light and noises on the photodiodes. The light source wavelengths are selected due to the minimum absorption coefficient of fat in these wavelengths. ${ }^{18}$ Two PIN photodiodes are used to measure intensity of scattered photons at the rate of $10 \mathrm{~Hz}$ which is suitable for the dynamic functional measurement of the tissue under test.

\section{Instrument Design}

The instrument uses a radio frequency light modulated DOS to measure real time optical properties of breast tissue. In order to properly perform the dynamic measurements of the vascular changes, the temporal resolution of the system is chosen at $5 \mathrm{~Hz}$ which is limited by the data acquisition and processing system. Four NIR light sources are employed to irradiate tissue in the instrument. The illuminated light penetrates the tissue and is scattered and absorbed while it is travelling toward the two photodiodes which are located in the reflectance geometry as shown in Fig. 1(a). Photodiodes \#1 and $\# 2$ are located $22.5 \mathrm{~mm}$ and $32.5 \mathrm{~mm}$ away from the light source, respectively. The scattered lights are detected by two PIN photodiodes and are amplified, filtered, and digitized by an impedance spectroscope; then the data are passed to the host computer for further analysis (Fig. 2(a)). A photograph of the designed radio frequency eLED light modulated DOS is presented in Fig. 1(b). 


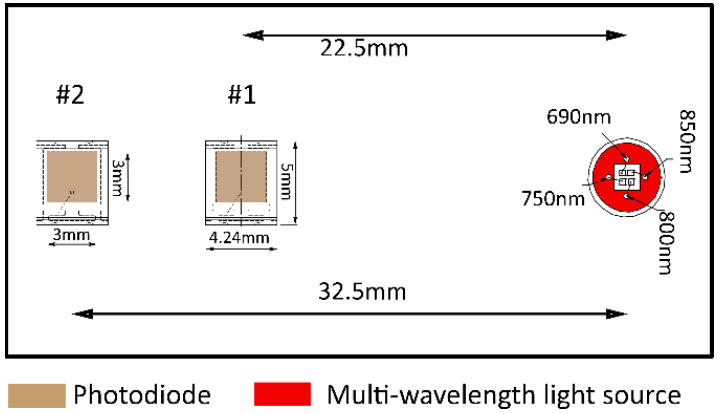

(a)

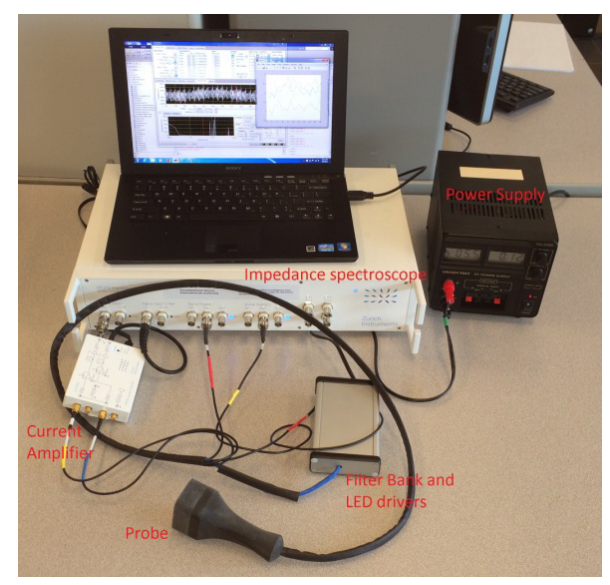

(b)

Fig. 1. (a) Source-detector configuration on the probe, and (b) Photograph of the radio frequency eLED light modulated DOS.

\subsection{Light illumination}

The system uses four wavelength NIR light sources at $690,750,800$, and $850 \mathrm{~nm}$, all generated by CW LEDs (Marubeni America Corporation L690/750/ $800 / 850)$. The wavelengths are chosen for the reason that the breast fat has a minimum absorption coefficient at the selected wavelengths ${ }^{18}$ which is the main constituent of the breast tissue (57\%). ${ }^{19}$ Therefore, maximum penetration occurs in the selected wavelengths. The light sources are encapsulated into a package and act as a point source in the system. The intensity of the LED with the wavelength of $690,750,800$ and $850 \mathrm{~nm}$ are modulated with 8, 10, 12 and $14 \mathrm{khz}$, respectively. An impedance spectroscope (Zurich Instruments Inc. HF2IS) is used to generate the required signals for the light sources, collect measured signals, process them, and pass the results to the host computer as shown in Fig. 2. A numerical oscillator generates sine and cosine signal pairs, which are used for the generation of the high-frequency output signals. A Mixer generates a weighted sum of generator outputs (maximum four) in order to generate the multi-frequency signal which is used to drive light sources. The HF2IS system was programmed to mix these four radio frequencies and make them available on one of the device's output (with 16-bit resolution Digital to Analog Convertor (DAC) and 210 Mega Sample per Second conversion rate). The

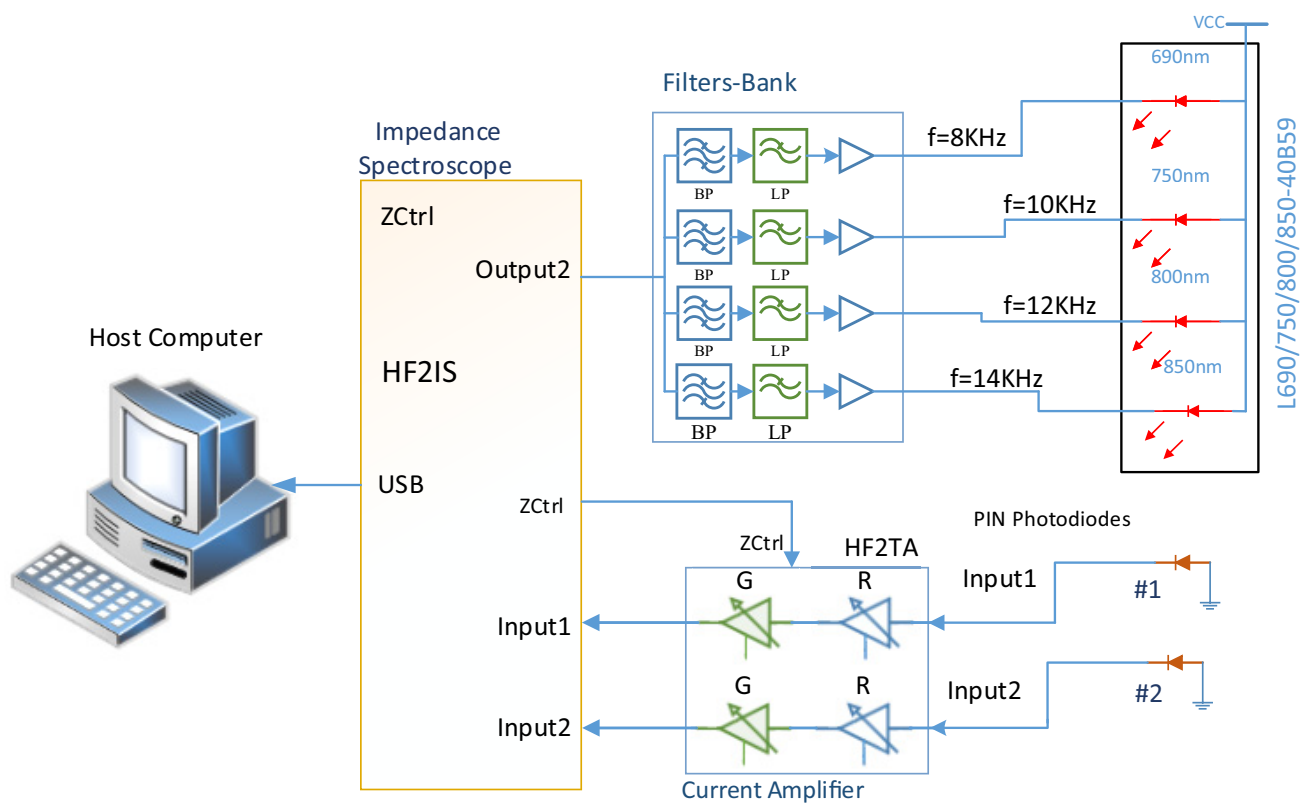

Fig. 2. Schematic block diagram of the system. 
amplitude of each individual frequency can be controlled and programmed through the host computer. In order to drive each LED with specific radio frequencies, the mixed output signal is filtered in a filter-bank to have each individual frequency available for light sources. For the reason that there are four light sources, four signal separation blocks are needed. Each block includes: a band-pass filter, a low pass filter, and an LED driver circuit. Each band-pass filter consists of a fourth-order switch capacitor Bessel filter (LTC1067-50, Linear Technology), with a center frequency of $8,10,12$, and $14 \mathrm{kHz}$. The separated sinusoidal signals are passed to the fourth-order low-pass Butterworth filter (LTC1563-2, Linear Technology) with a cut-of-frequency of $14 \mathrm{kHz}$ to remove some of the white noise and any unwanted signals which could affect the LEDs' signals (like the switching frequency of the band-pass filters). After purification of each radio frequency, they are passed into the driver circuit for the LED to modulate the intensity of the illuminated light. The maximum optical power incident was less than the maximum permissible exposure (MPE), defined by standards such as the "Safety of laser products-Part 1: Equipment classification and requirements". ${ }^{20}$ Using modulated light intensity sources has an important feature which allows for the simultaneous illumination of breast tissue with multiple wavelengths, and also improves the rejection of ambient light and noises.

\subsection{Light detection}

Scattered light is detected utilizing two PIN photodiodes (Vishay Semiconductor Inc. TEMD5010X01) that have high photosensitivity, high radiant sensitivity, and fast response times for NIR radiation which make it suitable for our application. The detected signals are amplified with a transimpedance amplifier (HF2TA) from Zurich Instruments Inc. as shown in Fig. 2. HF2TA includes two extremely low noise $(5 \mathrm{nV} / \sqrt{\mathrm{Hz}})$, independent programmable $50 \mathrm{MHz}$ amplification channels with a wide range of precise transimpedance gain selection options (100 V/A to $100 \mathrm{MV} / \mathrm{A}$ ) and very low current input leakage $(2 \mathrm{pA})$, which makes it entirely compatible for our needs. Each signal path in HF2TA has a high pass filter to remove low frequency components of the detected signals such as the DC component and line frequency noises, as well.

\subsection{Signal digitization and processing}

Following the HF2TA, the signals are passed to HF2IS and amplified into a pre-defined range, after which they are low pass filtered then digitized at a high speed rate (205 kilo sample per second) with a 14-bit Analog to Digital Convertor (ADC). The resulting digitized signals are then fed to the Digital Signal Processing (DSP) block with an ultra-high precision 128-bit DSP engine for demodulation. The ADC and DSP are configured to acquire 2048 samples for each measurement. The digitized data is then transmitted to the host computer via universal serial bus (USB2.0) in full speed configuration for further analyses. In order to extract intensity of each wavelength measured with photodiode \#1 and \#2, the collected data are passed through four digital band-pass filters in MATLAB ${ }^{\circledR}$ (Mathworks, Natick, Massachusetts) with a center frequency of $8,10,12$, and $14 \mathrm{khz}$. This technique allows removing all unwanted signals such as ambient light which has frequency around $120 \mathrm{~Hz}$ for fluorescent light and DC for tungsten bulb, and noises like white noises.

All collected data are quantified using an automated MATLAB ${ }^{\circledR}$ code to extract the concentrations of the four primary constituents including: oxyhemoglobin (cHb), deoxy-hemoglobin (cHbO2), water $(\mathrm{cH} 2 \mathrm{O})$, fat (cFat) and the average scattering coefficient of the medium, as well as concentration changes in $\mathrm{Hb}(\Delta \mathrm{cHb})$ and $\mathrm{HbO} 2(\Delta \mathrm{cHbO} 2)$ in the region of interest (ROI).

\section{Reconstruction Algorithm}

\subsection{Background}

In this section, light interaction with biological tissue and optical properties of breast tissue are briefly explained. Oxy-hemoglobin (HbO2), deoxy-hemoglobin $(\mathrm{Hb})$, fat, and water $(\mathrm{H} 2 \mathrm{O})$ are the four primary constituents in breast tissue, ${ }^{19,21}$ and the optical properties of these constituents are extensively studied and reported in, Refs. 8 and 23. These studies show that absorption of $\mathrm{HbO} 2, \mathrm{Hb}$, fat, and water are very low in the wavelength range of $650 \mathrm{~nm}-1000 \mathrm{~nm}$, which permits light to penetrate deeply into the breast tissue. ${ }^{8,9,24,25}$ The photons in biological tissue can consistently be modeled either numerically by Monte Carlo simulations or analytically by using the radiative transfer (RT) equation. A direct analytical solution for the RT equation is 
difficult in biological tissue, ${ }^{8}$ and most of the complications come from boundary conditions at the tissues surface. Since, in breast tissue, the scattering is sufficiently high and the absorption is very low in the NIR wavelength range $(650 \mathrm{~nm}-1000 \mathrm{~nm})$ the steady state RT equation can be approximated by the diffusion equation presented in (1). $8,9,24,25$

$$
\nabla D(r) \nabla \Phi_{d}(r)-\mu_{a} c_{m} \Phi_{d}(r)=-S(r),
$$

where $\Phi_{d}$ is the measured intensity at the sourcedetector separation of $d, S(r)$ represents a light source located at $r, \mu_{a}$ and $c_{m}$ are the absorption coefficient and velocity of photons in the medium, respectively, and $D$ is diffusion coefficient which is defined as below:

$$
\begin{gathered}
D=\left(c_{m}\left(3\left(\mu_{a}+(1-g) \mu_{s}\right)\right)^{-1},\right. \\
\mu_{t}^{\prime}=\mu_{a}+\mu_{s}^{\prime}=\mu_{a}+(1-g) \mu_{s},
\end{gathered}
$$

where $\mu_{t}^{\prime}, \mu_{s} \mu_{s}^{\prime}$, and $g$ are the attenuation coefficient, scattering coefficient, reduced scattering coefficient, and anisotropy, respectively. The diffusion approximation is valid for large source-detector separation $\left[r>10\left(\mu_{a}+\mu_{s}^{\prime}\right)-1\right]$ and where the absorption coefficient is much smaller than the scattering coefficient $\left(\mu_{a} \ll \mu_{s}^{\prime}\right) .{ }^{21}$ Equation (3) depicts the closed form analytic solution in spatially reflectance geometry for the diffusion equation in a semi-infinite homogeneous medium. ${ }^{11,26,29}$

$$
\begin{aligned}
R(d)= & \frac{1}{4 \pi \mu_{t}^{\prime}}\left[\left(\mu_{\mathrm{eff}}+\frac{1}{r_{1}}\right) \frac{\exp \left(-\mu_{\mathrm{eff}} r_{1}\right)}{r_{1}^{2}}\right. \\
& \left.+\left(\frac{4}{3} A+1\right)\left(\mu_{\mathrm{eff}}+\frac{1}{r_{2}}\right) \frac{\exp \left(-\mu_{\mathrm{eff}} r_{2}\right)}{r_{2}^{2}}\right] \\
r_{1}= & \sqrt{\left(\frac{1}{\mu_{t}^{\prime}}\right)^{2}+d^{2}}, \\
r_{2}= & \sqrt{\left(\frac{\left(\frac{4}{3}\right) A+1}{\mu_{t}^{\prime}}\right)^{2}+d^{2}}, \quad \mu_{\mathrm{eff}}=\sqrt{3 \mu_{a} \mu_{t}^{\prime}}
\end{aligned}
$$

where $\mu_{\text {eff }}$ is the effective attenuation coefficient, $R$ $(d)$ is light intensity measured at the distance of $d$ and $A$ is an internal reflection parameter that is referred to relative refractive index mismatch at airtissue interface. ${ }^{24}$

\subsection{Evaluation method}

The absorption coefficient and the reduced scattering coefficient of breast tissue are in the range of
$0.002 \mathrm{~mm}^{-1}-0.012 \mathrm{~mm}^{-1}$ and $0.6 \mathrm{~mm}^{-1}-1.5 \mathrm{~mm}^{-1}$, respectively, at the NIR wavelength window..$^{8,9,27}$ Therefore, $\mu_{t}^{\prime}$ value is in the range of $0.6 \mathrm{~mm}^{-1}$ $1.6 \mathrm{~mm}^{-1}$ and $\mu_{\text {eff }}$ value is between $0.05 \mathrm{~mm}^{-1}$ to $0.24 \mathrm{~mm}^{-1}$ for the breast tissue. Figure 3(a) shows the curve of $\log \left(r^{2} \times R\right)$ versus $\mu_{t}^{\prime}$ which refers to (3), while $\mu_{\text {eff }}$ is kept constant in the source-detection separation of $22.5 \mathrm{~mm}$ and $32.5 \mathrm{~mm}$. It depicts that variation of $\mu_{s}^{\prime}$ does not have remarkable variation on the back reflected signal at detector location. Also, the absorption coefficient of the breast tissue is much smaller than the reduced scattering coefficient, so fluctuation in absorption coefficient will not have noticeable variation on $\mu_{s}^{\prime}$. Thus, only changes on $\mu_{t}^{\prime}$ have an effect on the value of $\mu_{t}^{\prime}$. Troy et al. showed that there is no statistically

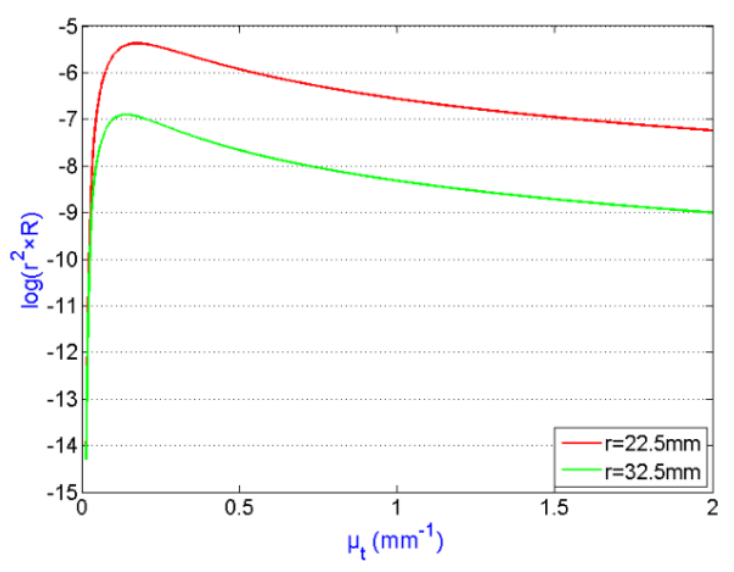

(a)

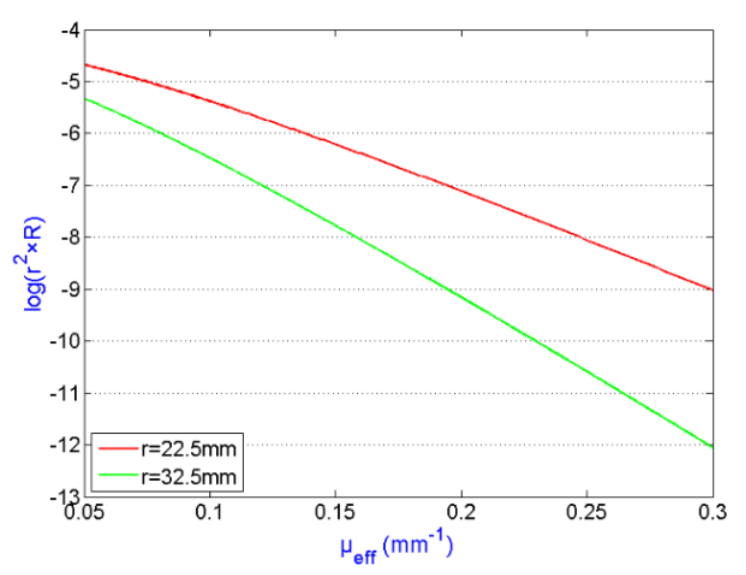

(b)

Fig. 3. (a) Plot of $\log \left(r^{2} \times R\right)$ versus $\mu_{t}^{\prime}$ while $\mu_{\text {eff }}=$ $1.7 \mathrm{~mm}^{-1}$, and (b) plot of $\log \left(r^{2} \times R\right)$ versus $\mu_{\text {eff }}$ while $\mu_{t}^{\prime}=1 \mathrm{~mm}^{-1}$ in the source-detection separation of $22.5 \mathrm{~mm}$ and $32.5 \mathrm{~mm}$. 
significant difference between the scattering coefficient of normal and diseased tissues. ${ }^{28}$ Garofalakis et al. have shown that measured mean values for the $\mu_{s}^{\prime}$ of the normal and the tumor tissue were $0.97 \pm 0.22 \mathrm{~mm}^{-1}$ and $1.08 \pm 0.18 \mathrm{~mm}^{-1}$, respectively. ${ }^{29}$ This variation does not cause remarkable variation on $\mu_{\text {eff }}$ but it changes $\mu_{s}^{\prime}$. According to Fig. 3(a), even if there were significant changes in $\mu_{t}^{\prime}$, there will be small changes in measured signal $\left(\log \left(r^{2} \times R\right)\right)$. Therefore, with a good approximation we can assume that $\mu_{t}^{\prime}$ is constant in the breast tissue.

Equation (3) demonstrates that fluctuations in $\mu_{s}^{\prime}$ and $\mu_{a}$ have considerable effect on $\mu_{\text {eff }}$. Figure

$$
\left[\begin{array}{l}
\mu_{a}(690 \mathrm{~nm}) \\
\mu_{a}(750 \mathrm{~nm}) \\
\mu_{a}(800 \mathrm{~nm}) \\
\mu_{a}(850 \mathrm{~nm})
\end{array}\right]_{r}=\left[\begin{array}{ll}
\varepsilon_{\mathrm{Hb}}(690 \mathrm{~nm}) & \varepsilon_{\mathrm{HbO}_{2}}(690 \mathrm{~nm}) \\
\varepsilon_{\mathrm{Hb}}(800 \mathrm{~nm}) & \varepsilon_{\mathrm{HbO}_{2}}(750 \mathrm{~nm}) \\
\varepsilon_{\mathrm{Hb}}(750 \mathrm{~nm}) & \varepsilon_{\mathrm{HbO}_{2}}(800 \mathrm{~nm}) \\
\varepsilon_{\mathrm{Hb}}(850 \mathrm{~nm}) & \varepsilon_{\mathrm{HbO}_{2}}(850 \mathrm{~nm})
\end{array}\right.
$$

3(b) shows the plot of $\log \left(r^{2} \times R\right)$ versus $\mu_{\text {eff }}$ which refers to (3), while $\mu_{t}^{\prime}$ is kept constant in the sourcedetection separation of $22.5 \mathrm{~mm}$ and $32.5 \mathrm{~mm}$. It shows that back reflected light at the detector location will dramatically change by a variation of $\mu_{\text {eff }}$. Since, there is no significant variation in $\mu_{s}^{\prime}$ for normal and diseased tissue, we can assume that there is a strong correlation between back reflected light and $\mu_{a}$. Four main chromophores contribute to the attenuation at wavelength $\lambda$ in breast tissue. The multi-spectral direct approach method is employed to extract these absorbers' concentration in two different path lengths for $r=22.5 \mathrm{~mm}$ and $r=32.5 \mathrm{~mm}$. where $\varepsilon$ and $C$ are the molar absorption extinction coefficient and total concentration of the chromophore in the light path. In different source-detector separation distance (in this study: $r=22.5 \mathrm{~mm}$ and $r=32.5 \mathrm{~mm}$ ), illuminated light travels through different path and depth in the tissue. In wider source-detector separation, measured light in the detector, travels through deeper and longer paths in the tissue. Concentrations of two absorbers vary by time: $\mathrm{HbO} 2$ and $\mathrm{Hb}$. Therefore, by solving (4), the total concentration of $\mathrm{Hb}(\mathrm{cHb}), \mathrm{HbO} 2$ (cHbO2), $\mathrm{H} 2 \mathrm{O}(\mathrm{cH} 2 \mathrm{O})$, fat (cFat), and concentration changes in $\mathrm{Hb}(\Delta \mathrm{cHb})$ and $\mathrm{HbO} 2(\Delta \mathrm{cHbO} 2)$ are calculated for two source-detector separation.

\section{Experimental Results and Discussion}

In order to evaluate the performance of the probe, an experimental study is performed on optical phantoms. A cylindrical shaped $(8 \mathrm{~cm}$ diameter and $10 \mathrm{~cm}$ length) acetal resin $\operatorname{rod}\left(\right.$ Delrin $\left.{ }^{\circledR}\right)$ is used to mimic background breast tissue which has similar optical properties to the breast fat $\left(\mu_{s}^{\prime}=2.3 \mathrm{~mm}^{-1}\right.$ and $\left.\mu_{a}=0.002 \mathrm{~mm}^{-1}\right) \cdot{ }^{30} \mathrm{~A}$ hole, with a $5 \mathrm{~mm}$ diameter, is put on the side wall of the cylinder, which is shown in Fig. 4, which is located $1.8 \mathrm{~cm}$ below one end of the acetal rod and is filled with tumor-like liquid phantom. A series of water, Intralipid ${ }^{\circledR} 20 \%$ emulsion (Fresenius Kabi Inc.) and black ink (Higgins India ink) solutions is used to

$$
\left.\begin{array}{ll}
\varepsilon_{\mathrm{H}_{2} \mathrm{O}}(690 \mathrm{~nm}) & \varepsilon_{\mathrm{Fat}}(690 \mathrm{~nm}) \\
\varepsilon_{\mathrm{H}_{2} \mathrm{O}}(750 \mathrm{~nm}) & \varepsilon_{\mathrm{Fat}}(750 \mathrm{~nm}) \\
\varepsilon_{\mathrm{H}_{2} \mathrm{O}}(800 \mathrm{~nm}) & \varepsilon_{\mathrm{Fat}}(800 \mathrm{~nm}) \\
\varepsilon_{\mathrm{H}_{2} \mathrm{O}}(850 \mathrm{~nm}) & \varepsilon_{\mathrm{Fat}}(850 \mathrm{~nm})
\end{array}\right] \times\left[\begin{array}{c}
C_{\mathrm{Hb}} \\
C_{\mathrm{HbO}_{2}} \\
C_{\mathrm{H}_{2} \mathrm{O}} \\
C_{\mathrm{Fat}}
\end{array}\right]_{r}
$$

mimic breast tumor in the background phantom. ${ }^{11}$ Intralipid ${ }^{\circledR}$ and Derlin have been chosen for its welldocumented optical properties and prior use in phantom studies to mimic optical properties of the breast tissue. ${ }^{30-34}$ Black ink is commonly used in phantom studies as an absorber due to its flat absorption spectrum in the NIR range and water-soluble properties.

In this research, two sets of phantom experiments were conducted to study the effect of changes of $\mu_{s}^{\prime}$ and $\mu_{a}$ on the measured light intensity. In the first experiment, 17 solutions of water and intralipid ${ }^{\circledR}$ with no added black ink were used to create inhomogeneity in the background phantom. Since, size of the hole is very small - in comparison to the path that light travels in the tissue to reach the photodiodes $\# 1$ and $\# 2-\mu_{s}^{\prime}$ will have a small variation. A reference solution of $3 \%(30 \mathrm{ml} / \mathrm{l})$ intralipid ${ }^{\circledR} 20 \%$ diluted in water was used. Then we increased the concentration of intralipid ${ }^{\circledR}$ from $30 \mathrm{ml} / \mathrm{l}$ to $50 \mathrm{ml} / \mathrm{l}$ in the step of $2 \mathrm{ml} / \mathrm{l}$ that increased the reduced scattering coefficient without any change in absorption coefficient. Figure 5 shows normalized back reflected absorption for varying concentrations of Intralipid ${ }^{\circledR}$ for source-detector separation of $22.5 \mathrm{~mm}$ and $32.5 \mathrm{~mm}$. As expected, fluctuation in $\mu_{s}^{\prime}$ does not have a measurable effect on the back reflected light at the detector location. Therefore, it has flat absorption within increasing intralipid ${ }^{\circledR}$ concentration. 


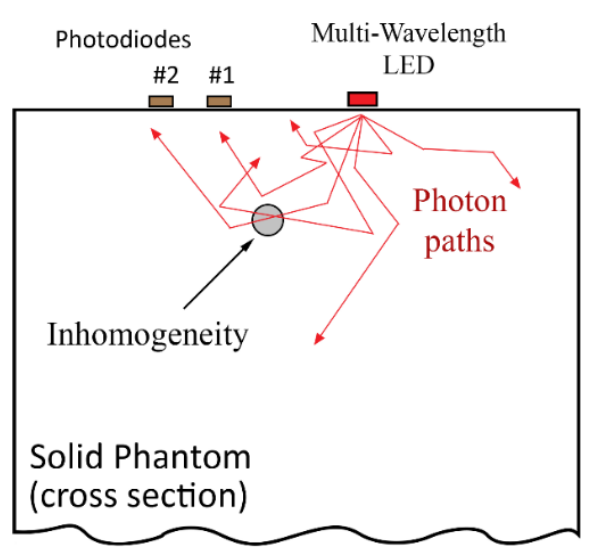

(a)

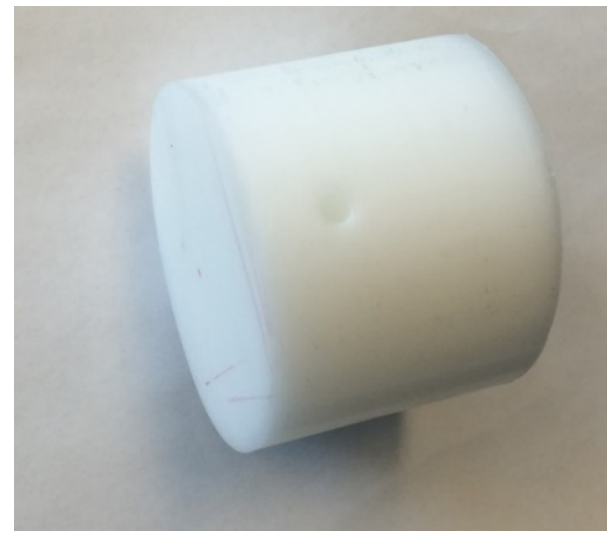

(b)

Fig. 4. The breast phantom constructed from acetal resin rod.

For the second experiment, a reference dilution of $50 \mathrm{ml} / 1$ intralipid ${ }^{\circledR}$ was used for calibration. In this case, the absorption coefficient increased by adding India black ink by $0.02 \mathrm{ml} / 1$ per step from $0.00 \mathrm{ml} / \mathrm{l}$ to $0.30 \mathrm{ml} / \mathrm{l}$ and the scattering coefficient of the medium was not affected by increasing the ink concentration. Figures 6(a) and 6(b) illustrate the reconstructed absorption coefficient of the phantom at location of photodiodes \#1 and \#2, respectively. The reconstructed $\mu_{a}$ for photodiode \#1 is smaller than photodiode \#2 because of the source-detector separation distance. In larger source-detector separation, illuminated photons travel deeper in tissue to reach the detector. Thus, the reconstructed value for $\mu_{a}$ has direct correlation with the depth of the tumor.

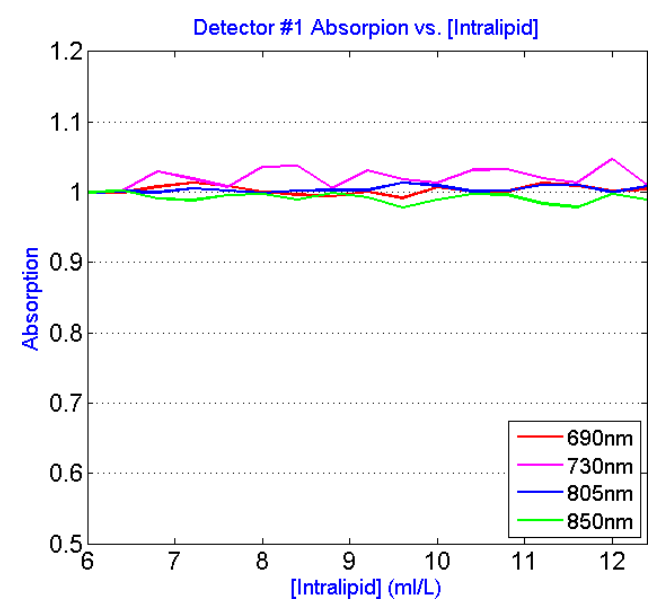

(a)
In order to assess performance of the probe to precisely separate different chromophores, such as $\mathrm{HbO} 2$, and $\mathrm{Hb}$, protein assay reagent (PAR) is used to create inhomogeneity inside the phantom which has similar optical properties to $\mathrm{HbO} 2$ in the NIR range except that it has a small absorbance shift. We used $1.5 \mathrm{~mL}$ Pierce $^{\mathrm{TM}} 660 \mathrm{~nm}$ PAR (Thermo ScientificTM Inc.) mixed with $10 \mu \mathrm{L}$ Pre-diluted protein assay standards: Bovine Serum Albumin (BSA), with $1000 \mu \mathrm{g} / \mathrm{mL}$ in $0.9 \%$ saline and $0.05 \%$ sodium azide to create a PAR. Figure 7 shows absorption coefficient spectrum of the PAR with BSA (RBSA) measured with Agilent Cary $60 \mathrm{UV}$-Vis spectrophotometer. It depicts that absorption coefficient of RBSA at $690 \mathrm{~nm}$ wavelength $\left(0.17 \mathrm{~mm}^{-1}\right)$ is much higher than its

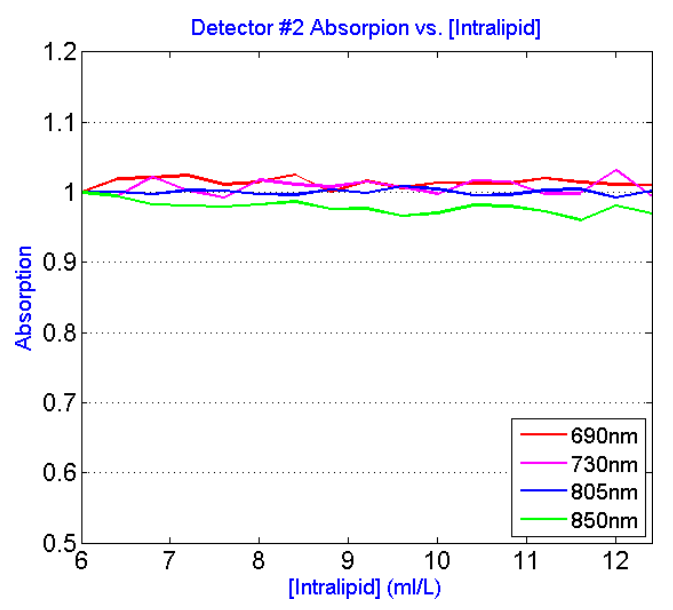

(b)

Fig. 5. Reconstructed value of absorption for linear increasing of intralipid ${ }^{\circledR}$ (a) at detector \#1 location, and (b) at detector \#2 location. 


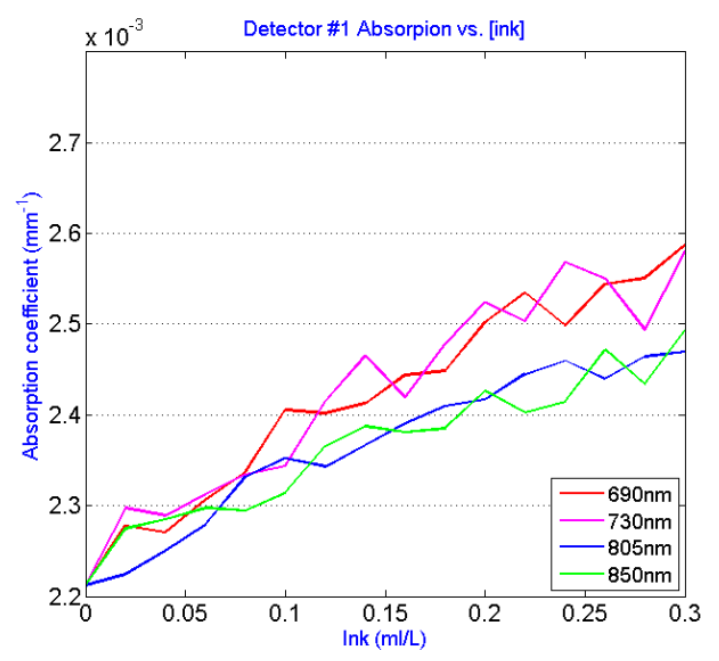

(a)

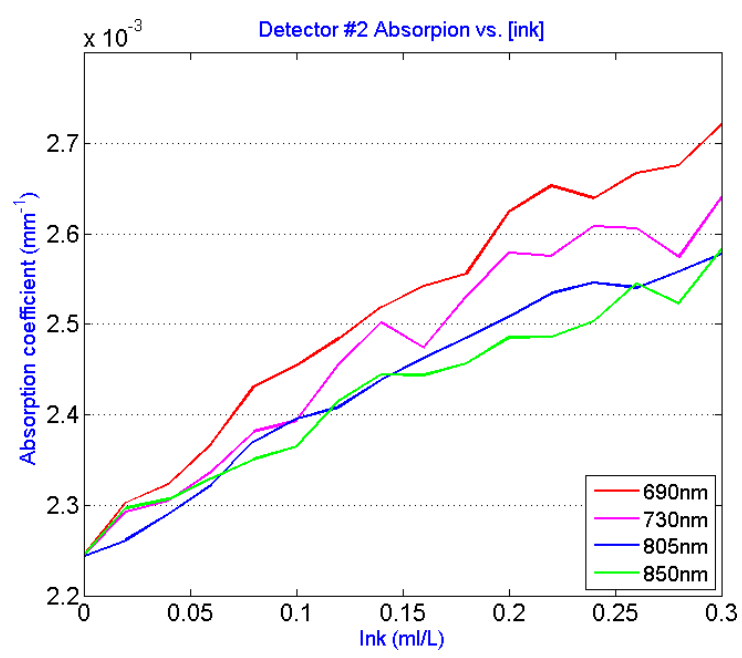

(b)

Fig. 6. Reconstructed absorption coefficient for linear increasing of India ink (a) at detector \#1 location, and (b) at detector \#2 location.

absorption coefficient at $750 \mathrm{~nm}, 800 \mathrm{~nm}$ and $850 \mathrm{~nm}\left(0.015 \mathrm{~mm}^{-1}\right)$.

The test results presented in Fig. 8, demonstrate absorption coefficient for reference phantom and phantom with RBSA inhomogeneity at four wavelengths. As we expected from Fig. 7, absorption coefficient of phantom with RBSA at $690 \mathrm{~nm}$ is higher than its absorption coefficient at $750 \mathrm{~nm}$, $800 \mathrm{~nm}$ and $580 \mathrm{~nm}$. Therefore, the probe can distinguish different chromophores which have different absorbance at the utilized wavelengths with sufficient accuracy. Figure 8 also shows that the measured absorption coefficient at the location of photodiode \#2 is higher than photodiode \#1 at the

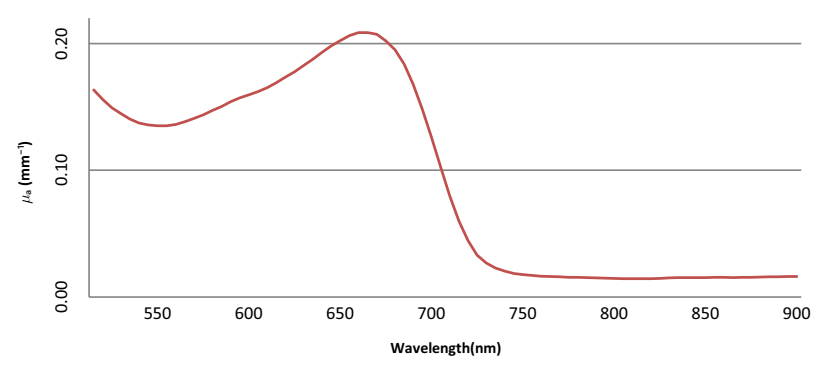

Fig. 7. Absorption coefficient spectrum of Pierce ${ }^{\mathrm{TM}} 660 \mathrm{~nm}$ PAR mixed with $10 \mu \mathrm{L}$ of $1000 \mu \mathrm{g} / \mathrm{mL}$ BSA.

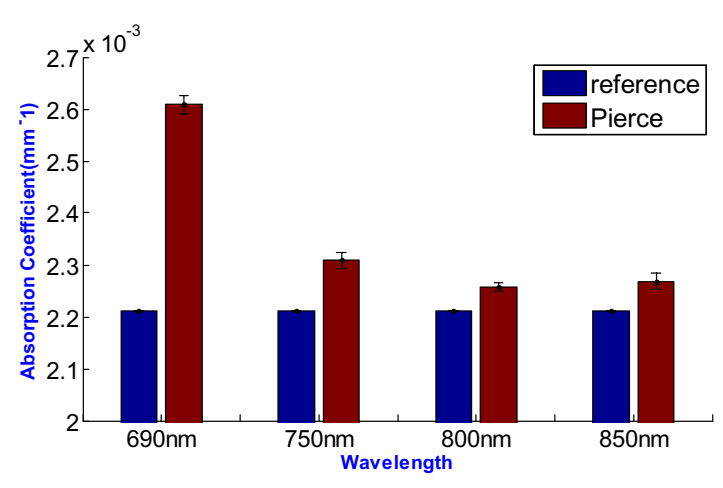

(a)

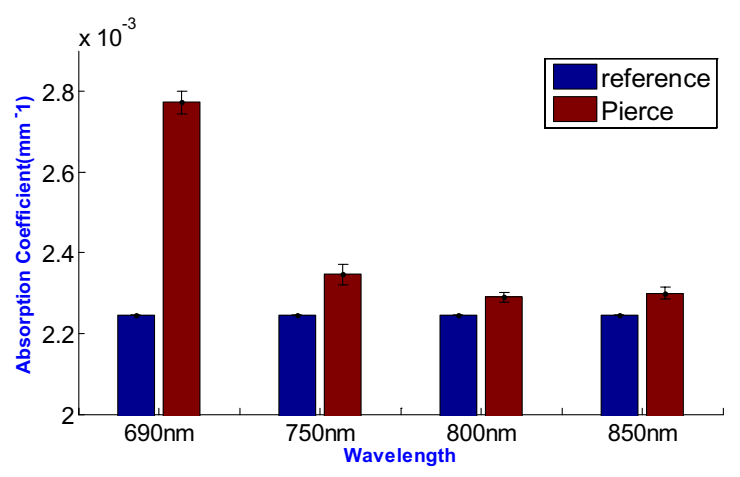

(b)

Fig. 8. Reconstructed absorption coefficient for reference phantom and phantom with Pierce ${ }^{\mathrm{TM}}$ PAR inhomogeneity (a) at detector \#1 location and (b) at detector \#2 location.

wavelength of $690 \mathrm{~nm}$ due to source-detector separation and the depth of the inhomogeneity.

\section{Conclusion}

We have presented a new handheld DOS probe for breast tissue assessment which employs radio frequency modulated DOS technique with four wavelengths encapsulated in an LED light source in the NIR. In this study we demonstrate that the 
proposed probe and method, accurately measure the optical properties of breast tissue-like phantoms and there is no need to use laser diodes or fiber coupled laser diodes as a light source. The device uses real time data acquisition system and the speed of data analyzing algorithm in the host computer is limited to $10 \mathrm{~Hz}$, which is sufficient for functional imaging of biological tissue. The device can be used for many existing clinical application which are involved in real-time measurement of tissue's optical properties and will create opportunities for new clinical applications including brain or limbs without any changes in hardware design. This research has now received ethical approval for clinical trials, and the device used herein has received the Health Canada Investigational Testing Authorization class II. In the next phase, therefore, the authors will apply this work to in-vivo studies on both healthy subjects and known breast cancer patients.

\section{Acknowledgments}

Financial support of this project was provided inpart by the Natural Sciences and Engineering Research Council of Canada (NSERC).

\section{References}

1. American Cancer Society, "Cancer Facts \& Figures 2015," (2015).

2. Canadian Cancer Society, "Special topic: Predictions of the future burden of cancer in Canada," (2015).

3. L. Tabár, P. B. Dean, "A new era in the diagnosis and treatment of breast cancer," Breast J. 16(Suppl 1), S2-S4 (2010).

4. H. D. Nelson, K. Tyne, A. Naik, C. Bougatsos, B. K. Chan, L. Humphrey, "Screening for breast cancer: Systematic evidence review update for the U. S. preventive services task force," Ann. Intern. Med. 151(10), 716-726 (2009).

5. A. B. de González S. Darby, "Risk of cancer from diagnostic X-rays: Estimates for the UK and 14 other countries," Lancet 363(9406), 345-351 (2004).

6. C. M. Ronckers, C. A. Erdmann, C. E. Land, "Radiation and breast cancer: A review of current evidence," Breast Cancer Res. 7(1), 21-32 (2005).

7. A. Hassan, M. El-Shenawee, "Review of electromagnetic techniques for breast cancer detection," IEEE Rev. Biomed. Eng. 4, 103-118 (2011).
8. T. Vo-dinh, Biomedical Photonics Handbook, CRC Press: Boca Raton, London, New York (2003).

9. D. A. Boas, C. Pitris, N. Ramanujam, Handbook of Biomedical Optics, CRC Press: Boca Raton, London, New York (2011).

10. L. V. Wang, H. Wu, Biomedical Optics: Principles and Imaging, Wiley-Interscience, John Wiley \& Sons Inc., Hoboken, New Jersey (2007).

11. M. L. Flexman, H. K. Kim, R. Stoll, M. A. Khalil, C. J. Fong, A. H. Hielscher, "A wireless handheld probe with spectrally constrained evolution strategies for diffuse optical imaging of tissue," Rev. Sci. Instrum. 83(3), 033108 (2012).

12. S. K. Biswas, K. Rajan, R. M. Vasu, "Diffuse optical tomographic imager using a single light source," $J$. Appl. Phys. 105(2), 024702 (2009).

13. S. J. Erickson, A. Godavarty, S. L. Martinez, J. Gonzalez, A. Romero, M. Roman, A. Nunez, J. Ge, S. Regalado, R. Kiszonas, C. Lopez-Penalver, "Handheld optical devices for breast cancer: Spectroscopy and 3-D Tomographic imaging," IEEE J. Sel. Top. Quantum Electron. 18(4), 1298-1312 (2012).

14. S. J. Erickson, A. Godavarty, "Hand-held based near-infrared optical imaging devices: A review," Med. Eng. Phys. 31(5), 495-509 (2009).

15. K. S. No, Q. Xie, R. Kwong, A. Cerussi, B. J. Tromberg, P. H. Chou, "HBS: A Handheld breast cancer detector based on frequency domain photon migration with full heterodyne," 2006 IEEE Biomedical Circuits and Systems Conf., pp. 114-117, Nov. 2006. Biomedical Circuits and Systems Conference, BioCAS 2006. IEEE, London.

16. H. Yang, L. Xi, S. Samuelson, H. Xie, L. Yang, H. Jiang, "Handheld miniature probe integrating diffuse optical tomography with photoacoustic imaging through a MEMS scanning mirror," Biomed. Opt. Express 4(3), 427-432 (2013).

17. A. Cerussi, N. Shah, D. Hsiang, A. Durkin, J. Butler, B. J. Tromberg, "In vivo absorption, scattering, and physiologic properties of 58 malignant breast tumors-determined by broadband diffuse optical spectroscopy," J. Biomed. Opt. 11(4), 044005 (2012).

18. M. Flexman, "Dynamic Digital Optical Tomography for Cancer Imaging and Therapy Monitoring," PhD Thesis, School of Arts and Science, Columbia University, 2012.

19. T. Durduran, R. Choe, J. P. Culver, L. Zubkov, M. J. Holboke, J. Giammarco, B. Chance, A. G. Yodh, "Bulk optical properties of healthy female breast tissue," Phys. Med. Biol. 47(16), 2847-2861 (2002).

20. IEC 60825-1, "Safety of laser products Part 1: Equipment classifcation and requirements," (2007).

21. F. Bevilacqua, A. J. Berger, A. E. Cerussi, D. Jakubowski, B. J. Tromberg, "Broadband 
absorption spectroscopy in turbid media by combined frequency-domain and steady-state methods," Appl. Opt. 39(34), 6498-6507 (2000).

22. S. A. Prahl, "Optical properties spectra," (2001), [Online], Available at: http://omlc.ogi.edu/spectra. [Accessed: 01-Sep-2013].

23. P. Taroni, A. Pifferi, A. Torricelli, D. Comelli, R. Cubeddu, "In vivo absorption and scattering spectroscopy of biological tissues," Photochem. Photobiol. Sci. 2(2), 124-129 (2003).

24. T. J. Farrell, "A diffusion theory model of spatially resolved, steady-state diffuse reflectance for the noninvasive determination of tissue optical properties in vivo," Med. Phys. 19(4), 879 (1992).

25. S. L. Jacques, B. W. Pogue, "Tutorial on diffuse light transport," J. Biomed. Opt. 13(4), 041302 (2008).

26. T. J. Farrell, B. C. Wilson, M. S. Patterson, "The use of a neural network to determine tissue optical properties from spatially resolved diffuse reflectance measurements," Phys. Med. Biol. 37(12), 22812286 (1992).

27. S. Fantini, A. Sassaroli, "Near-infrared optical mammography for breast cancer detection with intrinsic contrast," Ann. Biomed. Eng. 40(2), 398-407 (2012).

28. T. L. Troy, D. L. Page, E. M. Sevick-Muraca, "Optical properties of normal and diseased breast t issues: Prognosis for optical mammography," J. Biomed. Opt. 1(3), 342-355 (1996).
29. A. Garofalakis, G. Zacharakis, G. Filippidis, E. Sanidas, D. D. Tsiftsis, E. Stathopoulos, M. Kafousi, J. Ripoll, T. G. Papazoglou, "Optical characterization of thin female breast biopsies based on the reduced scattering coefficient," Phys. Med. Biol. 50(11), 2583-2596 (2005).

30. R. Berg, S. Andersson-Engels, O. Jarlman, S. Svanbrg, "Time-gated viewing studies on tissuelike phantoms," Appl. Opt. 35(19), 3432-3440 (1996).

31. S. T. Flock, S. L. Jacques, B. C. Wilson, W. M. Star, M. J. C. van Gemert, "Optical properties of intralipid: A phantom medium for light propagation studies," Lasers Surg. Med. 12(5), 510-519 (1992).

32. S. B. Colak, M. B. van der Mark, G. W. T. Hooft, J. H. Hoogenraad, E. S. van der Linden, F. A. Kuijpers, "Clinical optical tomography and NIR spectroscopy for breast cancer detection," IEEE J. Sel. Top. Quantum Electron. 5(4), 1143-1158 (1999).

33. H. J. van Staveren, C. J. Moes, J. van Marie, S. A. Prahl, M. J. van Gemert, "Light scattering in Intralipid-10\% in the wavelength range of 400-1100 nm," Appl. Opt. 30(31), 4507-4514 (1991).

34. Institut national d'optique, "Biomimic Optical Phantoms," (2015), [Online], available at: http:// www.ino.ca/en/products/. 